\title{
Multi-drug Resistant Citrobacter freundii Isolates in a Burn Hospital in Northeast of Iran: A Single-Center Cross-sectional Study
}

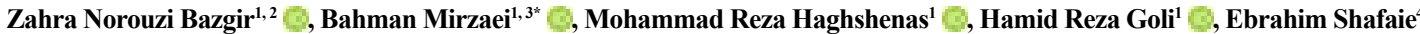 \\ 1. Department of Medical Microbiology and Virology, Faculty of Medicine, Mazandaran University of Medical Sciences Sari, Iran. \\ 2. Student Research Committee, Faculty of Medicine, Mazandaran University of Medical Sciences, Sari, Iran \\ 3. Department of Medical Microbiology and Virology, School of Medicine, Zanjan University of Medical Sciences, Zanjan, Iran. \\ 4. Infectious Diseases Research Center, Birjand University of Medical Sciences, Birjand, Iran
}

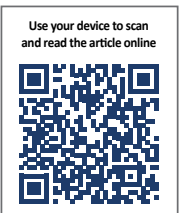

Citation Norouzi Bazgir Z, Mirzaei B, Haghshenas MR, Goli HR, Shafaie E. Multi-drug Resistant Citrobacter freundii Isolates in a Burn Hospital in Northeast of Iran: A Single-Center Cross-sectional Study. Research in Molecular Medicine. 2020; 8(2):63-70. https://doi.org/10.32598/rmm.8.2.893.1

https://doi.org/10.32598/rmm.8.2.893.1

(a) 0 (3)

Article Type:

Research Article

Article info:

Received: 05 Feb 2020

Revised: 10 Mar 2020

Accepted: 29 Mar 2020

Keywords:

Citrobacter freundii,

Clinical specimens,

Susceptibility testing

\begin{abstract}
A B S T R A C T
Background: Multi-drug resistant (MDR) Citrobacter freundii (C. freundii) as a causative agent of nosocomial infections is a health threat, especially in hospitals. This study was conducted to determine the prevalence of MDR C. freundii, considering isolation sites and a variety of utilized antibiotics.

Materials and Methods: In this cross-sectional study, the clinical samples of $C$. freundii strains were collected and screened using traditional bacteriological tests in Zareh Hospital, Sari City, Iran, during 2016-2017. We used disk diffusion methods to assess the susceptibility patterns of isolates according to the Clinical Laboratory Standard Institute (CLSI) guidelines.
\end{abstract}

Results: Out of 3248 clinical samples, $C$. freundii strains were detected in 109 samples $(32.1 \%$ females and $67.9 \%$ males). Susceptibility tests indicated that 89 isolates ( $81.65 \%$ ) were MDR strains. Frequencies of MDR C. freundii strains were higher in the Behavioral Intensive Care Unit (BICU) (37.61\%) and restoration ward (29.35\%) compared with other hospital wards.

Conclusion: Considering the MDR C. freundii strains detected from burn hospital wards, it is necessary to implement prevention criteria for their eradication from burn hospitals. The results indicate the urgent need to design more practical methods for controlling infection in hospital wards.

\section{Introduction}

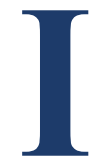

$\mathrm{n}$ recent years, nosocomial infections in burn hospitals have become one of the most common causes of mortality and morbidity. Gramnegative bacteria are the leading causes of mentioned infections [1-3]. The genus Citro- bacter belongs to a group of facultative anaerobic Gramnegative bacilli (from the Enterobacteriaceae family) frequently found in water, soil, and food [4]. Members of Citrobacter are also found infrequently as normal inhabitants of the intestinal tract of humans. Also, they are among the most prevalent bacteria isolated from nosocomial infections and known to play a role in the de-

\footnotetext{
* Corresponding Author:

Bahman Mirzaei, PhD.

Address: Department of Medical Microbiology and Virology, School of Medicine, Zanjan University of Medical Sciences, Zanjan, Iran.

Phone: +98 (912) 3397854

E-mail:dr.bahman.m@gmail.com
} 
velopment of bacteremia, meningitis, diarrhea, and brain abscess. However, their role as a cause of urinary tract infections in children has not been studied well. These organisms may also be responsible for severe infections, especially in immune-compromised hosts [5].

The genus of Citrobacter comprised 11 different species, among which $C$. freundii is the most prevalent found in infections. C. freundii is associated with opportunistic nosocomial infections such as Urinary Tract Infections (UTIs), wound infections, gastrointestinal diseases, septicemia, and meningitis, especially in immunecompromised patients [6-8]. Although rare in newborns, Citrobacter infection usually leads to meningitis and intracranial abscesses that in $75 \%$ of patients with meningitis associates with a high rate of mortality and morbidity $[5,9,10]$. The mortality rate for meningitis caused by Citrobacter spp. has been reported at about 30\% [11]. Citrobacter infections can be fatal, with $33 \%-48 \%$ overall death rates, and $30 \%$ in neonates [12].

In recent years, the emergence of Multi-Drug Resistant (MDR) Citrobacter to a wide range of antibiotic classes, including aminoglycosides, fluoroquinolones, and cotrimoxazole, has become a significant concern [13]. Multidrug-resistant mechanisms of Citrobacter strains have severely hampered our control efforts. Akya, in a study, showed that, out of 70 Citrobacter isolates, the highest rate of resistance belonged $(84.8 \%)$ to cefazolin, and cotrimoxazole (36.4\%), and the lowest to gentamicin (9.1\%) [9]. Cleaning and observing disinfection procedures are the main approaches to prevent nosocomial infections; otherwise, the rate of antibiotic-resistant bacteria would be continuously increased, making the treatment process problematic, resulting in a longer duration of hospitalization, increased treatment costs, and higher rates of mortality $[14,15]$. Therefore, the determination of resistance patterns among Citrobacter spp. would be of great medical value [9]. Given the overuse of antibiotics in Iran and a constant increase in antibiotic resistance, it is necessary to evaluate resistant strains via antibiotic susceptibility tests to prevent the emergence of new resistant strains [16].

Accordingly, regarding the undeniable role of $C$. freundii in the development of nosocomial infections in burn hospitals and its high resistance to several antibiotics, the present study was conducted to evaluate the prevalence of $C$. freundii and its antibiotic susceptibility pattern.

\section{Materials and Methods}

\section{Sample Collection and Isolation}

In this cross-sectional study (2016- 2017), a total of 3248 clinical samples, including wound, urine, sputum, blood, feces, and trachea, were isolated during one year from Zareh Burn Hospital, Sari City, Iran. The samples were grown on selective media, including blood agar, MacConkey agar, and thioglycollate broth based on bacteriology standards. Furthermore, blood samples were grown on TSB (Tryptic Soy Broth), and following 2448 hours of incubation at $37^{\circ} \mathrm{C}$, Gram-staining procedure was done to differentiate Gram-negative bacilli.

\section{Identification of Bacterial Isolates}

Bacterial identification of the isolates was performed according to conventional protocols mentioned in the Manual of Clinical Microbiology book [17]. In brief, biochemical tests, including oxidase, catalase, and motility detection in SIM medium, citrate, indole, methyl red, Voges-Proskauer, malonate utilization, H2S production lysine decarboxylase, and arginine dehydrogenase were done, and 109 samples were detected as having $C$. freundii. All of the utilized media were prepared as commercial media from HiMedia inc, India.

\section{Antibiotic Susceptibility Tests}

Isolates confirmed as $C$. freundii by biochemical tests underwent disk diffusion susceptibility test based on the CLSI protocol [18]. In short, following preparation of $0.5 \mathrm{McFarland}$ standards of each isolate, inoculation of the Mueller-Hinton agar plate with the test organism was carried out by streaking the swab in back and forth motions. Antimicrobial impregnated disks (BD BBLTM Sensi-DiscTM), including amikacin $(30 \mu \mathrm{g})$, ceftazidime $(30 \mu \mathrm{g})$, cephalexin $(30 \mu \mathrm{g})$, ciprofloxacin $(5 \mu \mathrm{g})$, imipenem $(10 \mu \mathrm{g})$, meropenem $(10 \mu \mathrm{g})$, gentamicin $(10 \mu \mathrm{g})$, tobramycin $(10 \mu \mathrm{g})$, co-trimoxazole $(25 \mu \mathrm{g})$, and nitrofurantoin $(10 \mu \mathrm{g})$ were put on the surface of the agar, and the plates were incubated for 24 hours at $37^{\circ} \mathrm{C}$. Following incubation, inhibition zone sizes to the nearest millimeter were measured using a ruler. Using published CLSI guidelines, susceptibility, or resistance of the organism to each tested drug was determined.

\section{Results}

In the present cross-sectional study, 109 isolated samples from outpatients of Zareh Burn Hospital were detected as C. freundii positive by biochemical tests. A total of 35 iso- 


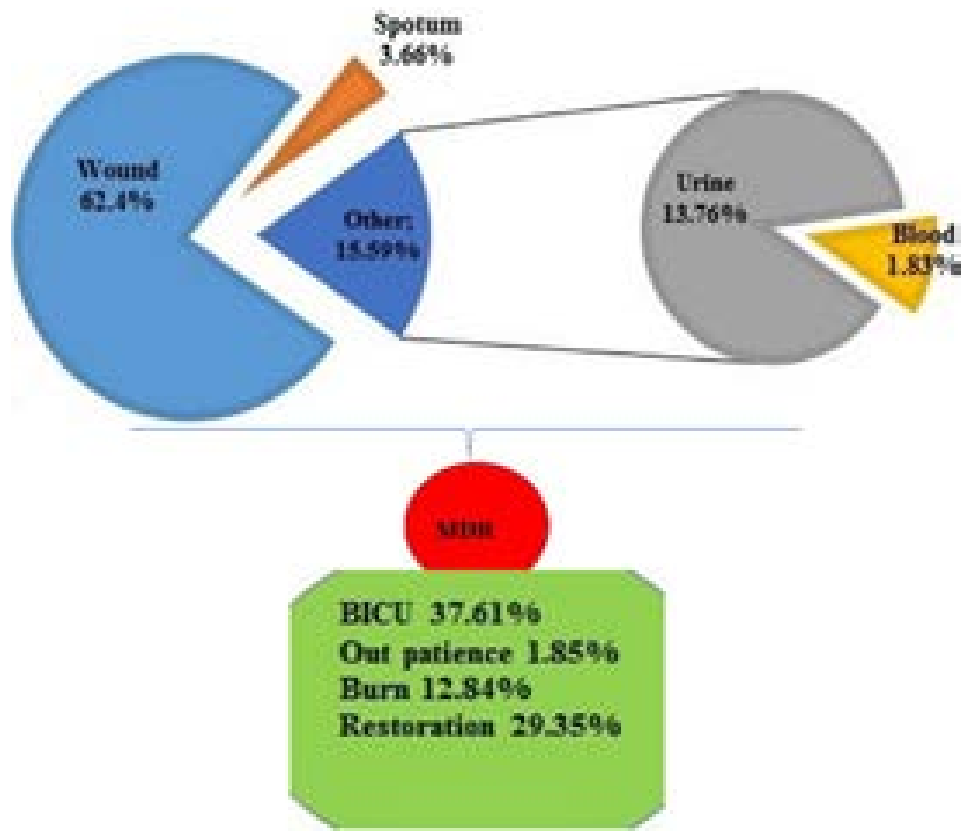

Figure 1. Frequency of MDR C. freundii isolates based on the clinical specimen and hospital wards

lates $(32.1 \%)$ belonged to females, and 74 isolates $(67.9 \%)$ belonged to males. Isolates were collected from the patients with a mean age of 49.7982 years. The eldest patient was 84 years old, while the youngest was only two years. Out of 109 obtained isolates, $79(72.5 \%)$ were from the wound, $21(19.3 \%)$ from urine, and $6(5.5 \%)$ from sputum and $3(2.7 \%)$ from the blood.
According to the results, in BICU, 41 isolates (37.61\%) were identified as MDR strains. A total of 32(29.35\%) were from the hospitals' restoration department; moreover, $40(36.69 \%)$ of BICU C. freundii isolates were detected among patients aged 21-70 years. Evaluation of the prevalence of antibiotic resistance showed that cephalexin (87.15\%), co-trimoxazole $(87.15 \%)$, and ciprofloxacin (79.81\%) possess the most strength. (Detailed data are list-

Table 1. Antimicrobial susceptibility pattern of (C. freundii) isolates

\begin{tabular}{|c|c|c|c|}
\hline \multirow{3}{*}{ Antibiotics } & \multicolumn{3}{|c|}{ Susceptibility Patterns } \\
\hline & \multicolumn{3}{|c|}{ No.(\%) } \\
\hline & Sensitive & Resistant & Intermediate \\
\hline Ciprofloxacin & $22(20.2)$ & $86(79.81)$ & $1(0.9)$ \\
\hline Cephalexin & $14(12.8)$ & $95(87.15)$ & 0 \\
\hline Ceftazidime & $25(22.93)$ & $81(74.31)$ & $3(2.8)$ \\
\hline Nitrofurantoin & $24(22.01)$ & $83(76.14)$ & $2(1.83)$ \\
\hline Amikacin & $52(47.7)$ & $53(48.6)$ & $4(3.7)$ \\
\hline Gentamicin & $22(20.2)$ & $87(79.8)$ & 0 \\
\hline Tobramycin & $33(30.3)$ & $75(68.8)$ & $1(0.9)$ \\
\hline Co-trimoxazole & $12(11)$ & $95(87.15)$ & $2(1.83)$ \\
\hline Imipenem & $32(29.35)$ & 77 (70.64) & 0 \\
\hline Meropenem & $33(30.3)$ & $76(69.7)$ & 0 \\
\hline
\end{tabular}


Table 2. Frequencies of isolated $C$. freundii according to the age, hospital wards, and specimens

\begin{tabular}{|c|c|c|c|c|c|c|c|c|c|}
\hline \multicolumn{6}{|c|}{ Hospital Wards } & \multicolumn{4}{|c|}{ Clinical Specimens } \\
\hline \multirow{2}{*}{ Age Range } & \multicolumn{9}{|c|}{ No. (\%) } \\
\hline & BICU, & $\begin{array}{c}\text { Out } \\
\text { patience }\end{array}$ & Burn & Restoration & Psychology & Wound & Urine & Sputum & Blood \\
\hline $0-10$ & $1(2.1)$ & $1(20)$ & - & - & - & $2(2.5)$ & - & - & - \\
\hline $11-20$ & $3(6.2)$ & - & $1(6.7)$ & - & - & $3(3.8)$ & - & - & $1(33.33)$ \\
\hline $21-30$ & 9 (18.8) & - & $1(6.7)$ & $4(10)$ & - & $9(11.4)$ & $2(9.5)$ & $2(33.3)$ & $1(33.33)$ \\
\hline $31-40$ & 9 (18.8) & - & $4(26.7)$ & $1(2.6)$ & - & $10(12.7)$ & 4 (19.15) & - & - \\
\hline $41-50$ & $5(10.4)$ & $2(40)$ & $2(13.35)$ & $9(23)$ & - & $13(16.4)$ & $5(23.85)$ & - & - \\
\hline $51-60$ & $10(20.8)$ & $1(20)$ & $5(33.3)$ & 4 (10.3) & $1(100)$ & $12(15.2)$ & $7(33.3)$ & 1 (16.7) & $1(33.33)$ \\
\hline $61-70$ & 7 (14.6) & - & $2(13.3)$ & $16(41)$ & - & $21(26.6)$ & $2(9.5)$ & $2(33.3)$ & - \\
\hline $71-80$ & $3(6.2)$ & - & - & $3(7.7)$ & - & $6(7.6)$ & - & - & - \\
\hline \multirow[t]{2}{*}{$81-90$} & $1(2.1)$ & $1(20)$ & - & $3(7.7)$ & - & $3(3.8)$ & $1(4.8)$ & $1(16.7)$ & - \\
\hline & $48(100)$ & $5(100)$ & $15(100)$ & 39 (100) & $1(100)$ & 79 (100) & $21(100)$ & $6(100)$ & $3(100)$ \\
\hline
\end{tabular}

Table 3. Percentage of MDR isolates based on the number of tested antibiotics

\begin{tabular}{|c|c|c|c|c|c|c|c|c|}
\hline \multirow{3}{*}{ Clinical Specimens } & \multicolumn{8}{|c|}{ Percentage of MDR Isolates to the Number of Antibiotics } \\
\hline & \multicolumn{8}{|c|}{ No.(\%) } \\
\hline & $\mathrm{R}$ to 10 & R to 9 & $\mathbf{R}$ to 8 & R to 7 & $\mathbf{R}$ to 6 & R to 5 & R to 4 & $\mathbf{R}$ to 3 \\
\hline Wound & $15(16.85)$ & $8(8.98)$ & $1(1.12)$ & $14(15.73)$ & $12(13.48)$ & $14(15.73)$ & $2(2.24)$ & $2(2.24)$ \\
\hline Urine & $7(7.86)$ & $4(4.49)$ & $2(2.24)$ & 0 & $1(1.12)$ & 0 & 0 & $1(1.12)$ \\
\hline Sputum & $22(2.24)$ & $22(2.24)$ & 0 & 0 & 0 & 0 & 0 & 0 \\
\hline Blood & $1(1.12)$ & 0 & $1(1.12)$ & 0 & 0 & 0 & 0 & 0 \\
\hline Trachea & 0 & 0 & 0 & 0 & 0 & 0 & 0 & 0 \\
\hline Stool & 0 & 0 & 0 & 0 & 0 & 0 & 0 & 0 \\
\hline
\end{tabular}

R: Resistant; 2-10: The number of resistant patterns to tested antibiotics. The percentage has been calculated based on the 109 C. freundii isolates.

Table 4. Frequencies of MDR isolates considering age, samples, and hospital wards

\begin{tabular}{|c|c|c|c|c|c|c|c|c|c|}
\hline \multicolumn{6}{|c|}{ Hospital Wards } & \multicolumn{4}{|c|}{ Clinical Specimens } \\
\hline \multirow{2}{*}{$\begin{array}{c}\text { Ages } \\
\text { Interval }\end{array}$} & \multicolumn{9}{|c|}{ No. (\%) } \\
\hline & BICU & Out Patience & Burn & Restoration & Psychology & Wound & Urine & Sputum & Blood \\
\hline $0-10$ & - & $1(50)$ & - & - & - & $1(1.5)$ & - & - & - \\
\hline $11-20$ & $2(4.9)$ & - & $1(7.1)$ & - & - & $2(3)$ & - & - & $1(50)$ \\
\hline $21-30$ & $8(19.5)$ & - & $1(7.1)$ & $3(9.7)$ & - & $8(11.9)$ & $2(13.3)$ & $2(50)$ & - \\
\hline $31-40$ & $7(17.1)$ & - & $3(21.5)$ & $1(3.2)$ & - & $9(13.4)$ & $2(13.3)$ & - & - \\
\hline $41-50$ & $5(12.2)$ & - & $2(14.3)$ & $7(22.5)$ & - & $11(16.4)$ & $3(20)$ & - & - \\
\hline $51-60$ & $10(24.4)$ & - & $5(35.7)$ & $2(6.5)$ & - & $10(14.9)$ & $5(33.4)$ & $1(25)$ & $1(50)$ \\
\hline $61-70$ & $6(14.6)$ & - & $2(14.3 \%)$ & $15(46.9)$ & - & $20(29.4)$ & $2(13.3)$ & $1(25 \%)$ & - \\
\hline $71-80$ & $3(7.3)$ & - & - & $2(6.5)$ & - & $5(7.5)$ & - & - & - \\
\hline $81-90$ & - & $1(50)$ & - & $2(6.5)$ & - & $2(3)$ & $1(6.7)$ & - & - \\
\hline Total & $41(100)$ & $2(100)$ & $14(100)$ & $32(100)$ & - & 68 (100) & 15 (100) & $4(100)$ & $2(100)$ \\
\hline
\end{tabular}


ed in Tables 1 and 2). Among all 109 samples isolated as C. freundii, 89 isolates $(81.65 \%)$ were reported as MDR. Table 2 presents the frequencies of $C$. freundii isolates.

As illustrated in Table 3, 4 and Figure 1, 68 (62.38\%) wound samples were MDR. Also, from wound and urine samples, $2(2.53 \%)$ and $3(14.28 \%)$ were susceptible to all tested antibiotics, respectively. The highest rates of antibiotic resistance in wound, urine, blood, and sputum were related to cephalexin, ciprofloxacin, tobramycin, and co-trimoxazole, in the descending order. Tables 3 and 4 presents detailed information.

\section{Discussion}

The prevalence of nosocomial infections caused by $C$. freundii, as an opportunistic pathogen in immune-compromised patients, has considerably increased over the past decade [9, 19]. Given the overuse of antibiotics in Iran, and constant rise in antibiotic resistance, evaluation of resistant strains by antibiotic susceptibility test seems to be critical to prevent the emergence of new resistant strains. In the present study, $C$. freundii isolates showed $47.7 \%$ susceptibility to amikacin, while in a study conducted in India, sensitivity to the mentioned antibiotic was reported as $53.4 \%$. Also, Jasemi, in a similar study, reported the susceptibility rate as $71.5 \%[6,20]$. Antibiotic resistance patterns not only differ in each country but also in each region due to genetic alteration and improper prescription of antibiotics [21].

In the current survey, $C$. freundii isolates demonstrated $70.64 \%$ susceptibility to imipenem, while, Akya reported a susceptibility rate of $100 \%$ for this bacterium to imipenem. Rate of resistance to carbapenems, as the last effective antibiotics against Citrobacter, has increased and become a primary concern worldwide, probably due to adopting improper infection control strategies in hospitals. Similar to the study conducted by Basavaraj in India, herein, most of $C$. freundii strains were isolated from the highest to the lowest from the wound, urine, body fluids, and blood samples $[6,9]$.

The mentioned isolates showed resistance to ciprofloxacin (79.81\%) and ceftazidime (74.31\%); however, in a study carried out in Kermanshah, Iran, resistance rate to mentioned antibiotics has been reported as $76.2 \%$ and $66.6 \%$, respectively. Consistent with the study by Jasemi, in the present research, amikacin and imipenem were the most effective antibiotics against $\mathrm{C}$. freundii infections.

Furthermore, the highest rate of resistance was reported for co-trimoxazole, ciprofloxacin, and ceftazidime [20].
Resistance to tobramycin (68.2\%), ceftazidime (76.4\%), amikacin (46.6\%), and gentamicin $(75.5 \%)$ were also reported, which was in line with results of a study carried out in 2014 (6). Over the past years, due to the overuse of antibiotics, the emergence of MDR C. freundii strains has occurred, making it a globally primary concern [13]. Resistance rates to cephalosporin were evaluated in this survey, and resistance to ceftazidime (74.31\%) and cefalexin $(87.15 \%)$ was also shown, while in previous studies, resistance to ceftazidime $(76.4 \%)$ and ceftriaxone $(40 \%)$ has been demonstrated [22]. Resistance to ceftazidime (86.3\%) has also been reported in a similar experiment by Jafari.

Moreover, resistance to fluoroquinolone, such as ciprofloxacin (79.81\%), has also been reported. Results of our survey showed that 2 out of $3 \mathrm{C}$. freundii isolates obtained from blood were MDR, which was consistent with the results of the study by Mahmoodi conducted in Hamadan, Iran [23]. In a study performed in Ethiopia, 3/4 of blood samples were reported positive for $C$. freundii [24]. Metri and his colleagues by conducting a retrospective study in 2013 to that patience with confirmed urine tract infection observed that Citrobacter isolates were found to be the third most common organism causing UTI in hospitalized patients. UTIs caused by Citrobacter species have been described in $5 \%$ to $12 \%$ of bacterial urine isolates in adults [6].

MDR Enterobacteriaceae isolates are responsible for creating a variety of infections in humans and animals. Considering the European Centre for Disease Prevention and Control (ECDC) and the Centers for Disease Control and Prevention (CDC), to create a standardized international terminology with which to describe acquired resistance profiles in PDR in Enterobacteriaceae are as follows: MDR: non-susceptible to $\geq 1$ agent in $\geq 3$ antimicrobial categories, and XDR (extensively-drug resistant): non-susceptible to $\geq 1$ agent in all but $\leq 2$ categories [25].

At the current research, 21 isolated $\mathrm{C}$. freundii were determined in the urine sample, and the prevalence of the mentioned bacterium from urine specimens was higher than other clinical specimens (except for wound specimens). In research conducted by Belachew in 2018, in Ethiopia, 11\% of isolated bacteria from river water samples were identified as C. freundii strains. Moreover, $60 \%$ of isolates were MDR C. freundii as well [26]. In 2011, Poirel was reported an isolated $\mathrm{C}$. freundii from an 18 years old cauterized patients that were highly resistant to $\beta$-lactams such as carbapenems, aminoglycosides, sulfonamides, tetracycline, and fluoroquinolones [26]. In this study, by conducting an antibiogram as a standard test for the evaluation of pheno- 
typic resistance to antibiotics out of 109 isolated C. freundii, 88 isolated were screened as MDR isolates according to the $\mathrm{CDC}$ and ECDC guidelines.

\section{Conclusion}

In the current study, the prevalence of MDR $C$. freundii was $81.65 \%$, highlighting the need for precise identification, eradication, and evaluation of antibiotic susceptibility pattern of this microorganism. The results call for the urgent design of more practical methods for infection control in hospital wards.

\section{Ethical Considerations}

\section{Compliance with ethical guidelines}

This study was approved by Mazandaran University of Medical Sciences ethics committee. All performed on the enlarged ethical statement IR.MAZUMS.REC1397.061 meeting number in Mazandaran University of Medical Sciences. In this study, all ethics including Ethics and Consent to participate from their parents have been collected in the research.

\section{Funding}

This research did not receive any specific grant from funding agencies in the public, commercial, or not-forprofit sectors.

\section{Authors contribution's}

Experimental tests: Zahra Norouzi Bazgir; Preparation of Manuscript and interperetation of results: Bahman Mirzaei; Writing the manuscript draft: Mohammad Reza Haghshenas; Experimental researches: Hamid Reza Goli; Final edditiing of manuscript: Ebrahim Shafaie

\section{Conflict of interest}

The authors declared no conflict of interest.

\section{Acknowledgements}

The authors wish to thank the Student Research Committee, Faculty of Medicine, Mazandaran University of Medical Sciences, Sari, Iran.

\section{References}

[1] Issler-Fisher AC, McKew G, Fisher OM, Harish V, Gottlieb T, Maitz PK. Risk factors for, and the effect of MRSA colonization on the clinical outcomes of severely burnt patients. Burns. 2015; 41(6):1212-20. [DOI:10.1016/j.burns.2015.03.003] [PMID]

[2] Hidalgo F, Mas D, Rubio M, Garcia-Hierro P. Infections in critically ill burn patients. Med Intensiva. 2016; 40(3):179-85. [DOI:10.1016/j.medin.2016.02.001] [PMID]

[3] Afkhamzadeh A, Majidi F, Ahmadi C. Risk factors for nosocomial infections among burn patients hospitalized in Tohid hospital, Sanandaj, Kurdistan Iran. Med J Mashhad Univ Med Sci. 2016; 59(4):225-32.

[4] Liu L-H, Wang N-Y, Wu AY-J, Lin C-C, Lee C-M, Liu C-P. Citrobacter freundii bacteremia: Risk factors of mortality and prevalence of resistance genes. J Microbiol, Immuno Infect. 2018; 51(4):565-72. [DOI:10.1016/j.jmii.2016.08.016] [PMID]

[5] Lavigne J-P, Defez C, Bouziges N, Mahamat A, Sotto A. Clinical and molecular epidemiology of multidrug-resistant Citrobacter spp. infections in a French university hospital. Eur J Clin Microbiol Infect Dis. 2007; 26(6):439-41. [DOI:10.1007/s10096-0070315-3] [PMID]

[6] Metri BC, Jyothi P, Peerapur BV. Antimicrobial resistance profile of Citrobacter species in a tertiary care hospital of Southern India. Indian J Med Sci. 2011; 65(10). [DOI:10.4103/00195359.109259] [PMID]

[7] Gill MA, Schutze GE. Citrobacter urinary tract infections in children. Pediatr Infect Dis J. 1999; 18(10):889-92. [DOI:10.1097/00006454-199910000-00010] [PMID]

[8] Leski TA, Taitt CR, Bangura U, Ansumana R, Stenger DA, Wang $\mathrm{Z}$, et al. Finished genome sequence of the highly multidrug-resistant human urine isolate Citrobacter freundii strain SL151. Genome Announc. 2016; 4(6):e01225-16. [DOI:10.1128/ genomeA.01225-16] [PMID] [PMCID]

[9] Akya A, Jafari S, Ahmadi K, Elahi A. Frequency of blaCTX-M, blaTEM and blaSHV genes in Citrobacters isolated from Imam Reza Hospital in Kermanshah. J Mazandaran Univ Med Sci. 2015; 25(127):65-73.

[10] Plakkal N, Soraisham AS, Amin H. Citrobacter freundii brain abscess in a preterm infant: A case report and literature review. Pediatr Neonatol. 2013; 54(2):137-40. [DOI:10.1016/j.pedneo.2012.10.004] [PMID]

[11] McPherson C, Gal P, Ransom JL. Treatment of Citrobacter kosen Infection with Ciprofloxacin and Cefotaxime in a Preterm Infant. Ann Pharmacother. 2008; 42(7-8):1134-8. [DOI:10.1345/ aph.1L008] [PMID]

[12] Pepperell C, Kus JV, Gardam MA, Humar A, Burrows LL. Low-virulence Citrobacter species encode resistance to multiple antimicrobials. Antimicrob Agents Chemother. 2002; 46(11):3555-60. [DOI:10.1128/AAC.46.11.3555-3560.2002] [PMID] [PMCID]

[13] Chen S, Hu F, Liu Y, Zhu D, Wang H, Zhang Y. Detection and spread of carbapenem-resistant Citrobacter freundii in a teaching hospital in China. Am J Infect Control. 2011; 39(9):e55-e60. [DOI:10.1016/j.ajic.2011.02.009] [PMID]

[14] Gastmeier P, Schwab F, Bärwolff S, Rüden H, Grundmann H. Correlation between the genetic diversity of nosocomial path- 
ogens and their survival time in intensive care units. J Hosp Infect. 2006; 62(2):181-6. [DOI:10.1016/j.jhin.2005.08.010] [PMID]

[15] Anusha S, Vijaya L, Pallavi K, Manna P, Mohanta G, Manavalan R. An Epidemiological study of surgical wound infections in a surgical unit of tertiary care teaching hospital. Indian J Pharm Pract. 2010; 3(4):8-13.

[16] Nikoonejad A, Gharabaghi N, Davari M, Ayremloo M, Nejad Rahim R. Sensitivity pattern and resistance against antibiotics in isolated microorganisms of hospitalized patients. J Urmia Univ Med Sci. 2013; 24(10):785-90.

[17] Schmidt HE, Montag MO, Bockemühl J, Heesemann JU, Karch HE. Shiga-like toxin II-related cytotoxins in Citrobacter freundii strains from humans and beef samples. Infect Immun. 1993; 61(2):534-43. [PMID] [PMCID]

[18] Doran TI. The role of Citrobacter in clinical disease of children. Clin Infect Dis. 1999; 28(2):384-94. [DOI:10.1086/515106] [PMID]

[19] Jasemi SS, Alipoor F, Dehbashi S, Mardaneh J. Isolation of Citrobacter spp. from Blood Specimens in Patients Hospitalized in Kermanshah Imam Khomeini hospital and determination of the of isolates sensitivity to antibiotics: Short Communication. J Birjand Univ. 21(3): 394-400.

[20] Mohammadi S, Mohammadi B, Zandi S, Ramazanzadeh R Rouhi S. Antibiotic Sensitivity In Strains Of Klebsiella Pneumonia Isolated From Clinical Samples Besat Hospitals Of Sanandaj (2013-2014). Zanko J Med Sci. 2016; 17(52):1-9.

[21] Kim PW, Harris AD, Roghmann M-C, Morris JG, Strinivasan A, Perencevich EN. Epidemiological risk factors for isolation of ceftriaxone-resistant versus-susceptible Citrobacter freundii in hospitalized patients. Antimicrob Agents Chemother. 2003; 47(9):2882-7. [DOI:10.1128/AAC.47.9.2882-2887.2003] [PMID] [PMCID]

[22] Mahmoudi H, Ghasemi Bassir HR, Hosseini SM, Arabestani MR, Alikhani MY. The frequency of bacteria isolated from blood cultures and antibiotic susceptibility patterns among admitted patients in Hospital of Hamedan University of Medical Sciences. Iran J Med Microbiol. 2016; 10(4):69-74.

[23] Zenebe T, Kannan S, Yilma D, Beyene G. Invasive bacterial pathogens and their antibiotic susceptibility patterns in Jimma University specialized hospital, Jimma, Southwest Ethiopia. Ethiop J Health Sci. 2011; 21(1):1-8. [DOI:10.4314/ejhs. v21i1.69038] [PMID] [PMCID]

[24] Magiorakos AP, Srinivasan A, Carey RB, Carmeli Y, Falagas ME, Giske CG, Harbarth S, Hindler JF, Kahlmeter G, OlssonLiljequist B, Paterson DL. Multidrug-resistant, extensively drug-resistant and pandrug-resistant bacteria: an international expert proposal for interim standard definitions for acquired resistance. Clin Microbiol Infect. 2012; 18(3):268-81. [DOI:10.1111/j.1469-0691.2011.03570.x] [PMID]

[25] Belachew T, Mihret A, Legesse T, Million Y, Desta K. High level of drug resistance by gram-negative bacteria from selected sewage polluted urban rivers in Addis Ababa, Ethiopia. BMC Res Notes. 2018; 11(1):524. [DOI:10.1186/s13104-018-3622-0] [PMID] [PMCID]

[26] Poirel L, Ros A, Carricajo A, Berthelot P, Pozzetto B, Bernabeu S, Nordmann P. Extremely drug-resistant Citrobacter freundii isolate producing NDM-1 and other carbapenemases identified in a patient returning from
India. Antimicrob Agents Chemother. 2011; 55(1):447-8. [DOI:10.1128/AAC.01305-10] [PMID] [PMCID] 
This Page Intentionally Left Blank 